\title{
Im Dialog über CED, Wissen auffrischen und mit Humor den Alltag würzen
}

\author{
Medizin interdisziplinär FOKUS \\ Interdisziplinäre Betrachtungen zur chronisch-entzündlichen Darmerkrankung
}

Interviewteilnehmer:

\section{Georg Kähler, ${ }^{a}$ Ulrich Böcker, ${ }^{b}$ Robert Ehehalt, ${ }^{c}$ Martin Götz, ${ }^{d}$ Peter Hasselblatt, ${ }^{e}$ Martin E. Kreis, ${ }^{f}$ Anton J. Kroesen, ${ }^{\mathrm{g}}$}

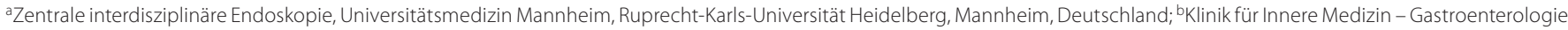
Diabetologie und Hepatologie, Vivantes Klinikum Neukölln, Berlin, Deutschland; ' Praxis für Innere Medizin und Gastroenterologie, Heidelberg, Deutschland; ${ }^{\mathrm{d}}$ Medizinische Klinik IV Gastroenterologie/Onkologie, Klinikum Sindelfingen-Böblingen, Böblingen, Deutschland; eKlinik für Innere Medizin II - Gastroenterologie, Hepatologie, Endokrinologie und Infektiologie, Universitätsklinikum Freiburg, Freiburg, Deutschland; ${ }^{\text {FKl } l i n i k ~ f u ̈ r ~ A l l g e m e i n-, ~ V i s z e r a l-~ u n d ~ G e f a ̈ ß c h i r u r g i e, ~ C h a r i t e ~-~ U n i v e r s i t a ̈ t s m e d i z i n ~ B e r l i n, ~ C a m p u s ~ B e n j a m i n ~ F r a n k l i n, ~ B e r l i n, ~}$ Deutschland; ${ }^{9}$ Allgemein-Viszeral- und Unfallchirurgie, Krankenhaus Porz am Rhein, Köln, Deutschland

Frage 1: Was ist die beste Methode für die Beobachtung von Patienten mit chronisch-entzündlicher Darmerkrankung (CED)? Sonografie, Endoskopie, Labor oder einfach klinisch?

Böcker: Das beste Mittel für die Tumor-Surveillance ist die Endoskopie, für die Überwachung auf Strikturen sind es die klinischen Symptome, für die Beobachtung des (distalen) Entzündungsgeschehens ist es das Calprotectin im Stuhl.

Ehehalt: Der Goldstandard ist wohl die Endoskopie, aus praktischen Gründen liegt aber die klinische Untersuchung in Verbindung mit Laborwerten vorn.

Götz: Tumor-Surveillance: Endoskopie; Ansprechen auf eine Behandlung: klinisch; Labor: häufig; Sonografie: wird geprüft, wahrscheinlich gut (Darmwanddicke, Limberg-Score); Endoskopie: optimal, aber invasiv und besonderen Situationen vorbehalten.

Hasselblatt: Der beste Ansatz zur routinemäßigen Beobachtung von Patienten mit CED ist klinisch in Kombination mit Calprotectin (Wert sollte <250 sein). Bei Symptomen nehmen wir Ultraschall hinzu, Endoskopie wenn nötig (oder indiziert; z.B. zur Tumor-Surveillance und wenn der Befund die Therapieentscheidung beeinflusst), gegebenenfalls auch MRT.

Kreis: Jede davon in angemessenen Intervallen.

Kroesen: Das hängt vom Zustand des jeweiligen Patienten ab. Im Allgemeinen sind Sonografie und klinische Untersuchung ausreichend. Wenn der Patient plötzlich schlechter wird oder zunehmend stenotische Beschwerden hat, kann eine Endoskopie erforderlich werden, vor allem wenn eine Operation oder endoskopische Dilatation als Behandlungsoption näherrückt.

Frage 2: Anastomotische Strikturen - Wie oft und mit welchem Durchmesser nehmen Sie Dilatationen vor oder empfehlen Sie die Re-Anastomosierung?

Böcker: Wenn die Striktur von begrenztem Umfang ist und der Patient zustimmt, immer erst endoskopisch behandeln, mit fraktio- nierter Ballondilatation, bevor man operiert. So oft wie notwendig wiederholen, bis ein durchschnittlicher Durchmesser von $15 \mathrm{~mm}$ erreicht ist.

Ehehalt: Bis maximal $3 \mathrm{~cm}$ Länge der Stenose: Dilatation auf max. $2 \mathrm{~cm}$.

Götz: 15 oder 18 mm (je nach verbleibender Lumenweite). Wiederholung je nach initialem Ansprechen (IA): gutes IA - so oft wie notwendig; mäßiges IA: Operation erwägen; kein IA: Operation.

Hasselblatt: Wir dilatieren in der Regel auf 20 mm (natürlich abhängig vom vorhandenen Durchmesser). Bei aktueller Inflammation geben wir zusätzlich eine antiinflammatorische Therapie bzw. erhöhen deren Dosis. Wenn die Symptome persistieren oder wiederkehren, werden erneut Untersuchungen angesetzt. Ansonsten streben wir eine endoskopische Kontrolle (mit Dilatation) nach 3-6 Monaten an, um Durchgängigkeit sicherzustellen (und nach Möglichkeit auf Mukosaheilung zu kontrollieren). Planmäßige wiederholte Dilatationen (wie bei Ösophagusstrikturen) machen wir nicht.

Kreis: Da gibt es keine festen Werte. Wir entscheiden das danach, wie lange der Erfolg der Dilatation anhält. Meist ist der Durchmesser $18 \mathrm{~mm}$.

Kroesen: Die Grundkriterien für die endoskopische Dilatation müssen erfüllt sein: Länge unter $3 \mathrm{~cm}$, keine Fisteln, kein Kinking oral der Striktur. Der Patient muss über die Vor- und Nachteile der endoskopischen Dilatation und chirurgischen Resektion aufgeklärt werden und seine Einwilligung geben; seine Entscheidung ist sehr wichtig. Patienten, die im Hinblick auf eine Operation unentschlossen sind, können durch eine Dilatation psychologisch darauf vorbereitet werden, wenngleich der Effekt der Dilatation nicht von langer Dauer ist.

Frage 3: Wie viele CED-Operationen pro Jahr muss man als Zentrum durchführen?

Böcker: 25.

Ehehalt: 10 Pouches. 
Götz: 20.

Hasselblatt: Ich würde sagen >50 CED-assoziierte chirurgische Eingriffe und $>10$ Proktokolektomien. Die Eingriffe sollen nach Möglichkeit laparoskopisch erfolgen.

Kreis: 300 abdominalchirurgische Eingriffe.

Kroesen: Zur Colitis ulcerosa gibt es zwei Studien. In beiden steht, pro Jahr sollten mindestens 10 Proktokolektomien mit ileoanalem Pouch durchgeführt werden. Das ist die minimal akzeptable Menge. Für Morbus Crohn lässt sich ein ähnlicher Wert für die entsprechenden Eingriffe postulieren. Aufgrund der großen Heterogenität des Krankheitsbildes ist es hier jedoch schwieriger, eine konkrete Zahl zu nennen. Vom Gefühl her würde ich sagen: mehr als 50 große abdominalchirurgische Eingriffe.

\section{Frage 4: Wie lauten die Kriterien für das primäre Versagen einer medikamentösen Therapie? Kann eine Operation zu früh kommen?}

Böcker: Versagen bedeutet, dass das gesetzte Therapieziel in einer für die jeweilige Medikation spezifischen Zeit nicht erreicht wird. Die Kriterien hängen davon ab, welches klinische, endoskopische oder sonstige Ziel im Rahmen der Treat-to-Target-Diskussion vor Therapiebeginn ausgewählt wurde. Eine Operation kann zu früh kommen, wenn der postoperative anatomische und funktionelle Status erhebliche Morbidität für den Patienten bedeuten und seine Lebensqualität einschränken könnte, obwohl dies bei realistischer Chancen/Risiko-Abwägung durch eine intensivere medikamentöse Therapie hätte vermieden werden können; Beispiel Resektion eines langen Dünndarmsegments.

Ehehalt: Ich verstehe die Frage nicht. Meinen Sie Versagen einer Steroid-Therapie? Einer Biologika-Therapie? Eine hohe fibrostenotische Stenose wäre natürlich ein Kriterium.
Götz: Klinisches Nichtansprechen. Eine Operation kann sowohl zu früh als auch zu spät kommen. Zu frühe Operation: hängt vom Umfang der Resektion ab (z.B. bei Proktokolektomie) - je größer der Umfang, desto mehr zögere ich (und zögern die Patienten), die Operation vorzunehmen.

Hasselblatt: Primäres Versagen bedeutet, dass innerhalb von 8-10 Wochen keine klinische/endoskopische Verbesserung eintritt. Die Kriterien sind abhängig von der initialen Symptomatik. Eine Operation bedeutet kein Therapieversagen, sondern kann eine sehr sinnvolle Alternative zur medikamentösen Therapie sein, wenn sie mit postoperativer Prophylaxe verknüpft wird. Sie sollte auf jeden Fall erwogen werden, wenn Biologika versagen. Bei Versagen einer konventionellen Therapie kann die Operation eine sinnvolle Alternative zu Anti-TNF darstellen.

Kreis: Persistierende Symptome, hohe Leukozyten-, CRP- und Calprotectin-Werte, Inflammation in der Bildgebung (Sonografie, Endoskopie, MRT).

Kroesen: Nur bei einem Patienten, der bisher keinerlei medikamentöse Therapie erhalten hat, kann eine Operation zu früh kommen. Sobald die Hauptindikationen gegeben sind, ist auch eine Operation indiziert. Im Hinblick auf Therapieversagen als Indikationsfaktor sollte fortgesetzte Kortikosteroidanwendung plus Biologika oder Einleitung einer Biologika-Drittlinientherapie als spätester Zeitpunkt für eine Operation betrachtet werden.

Übersetzung aus Visc Med 2019;35:359-361, DOl: 10.1159/000504102

\section{Blickfang Pathogenese FokUs}

\section{Chronisch-entzündliche Darmerkrankungen}

Im gesunden Darm sind sowohl kommensale als auch pathogene Bakterien in der gesamten der Mukosa angrenzenden Schleimschicht und im Darmlumen verteilt, während das Darmepithel intakt ist und daher resistent gegen eine bakterielle Invasion. Kommensale Mikrobiota und durch diese produzierte Substanzen (SCFA, Sphingolipide, Fragellin, PAMPs) beeinflussen die Enterozyten, die strukturelle und funktionelle Integrität der Schlussleisten und die mukosale Immunantwort positiv.

Bei Patienten mit CED führt eine durch verschiedene Faktoren verursachte Störung der Darmmikrobiota zu einem Ungleichgewicht in der Zusammensetzung der Darmmikrobiota (Dysbiose). Bakterien heften sich durch eine instablie und dünnere Schleimschicht vermehrt an das Darmepithel an und passieren die Schlussleisten leichter. Darüber hinaus entziehen dysbiotische Mikrobiota durch eine veränderte Produktion von SCFA, Sphingolipiden und Fragelin den Enterozyten die notwendige Energieversorgung und erschwe- ren dadurch eine Regeneration, stören die Integrität der Schlussleisten und führen schließlich zu einer Dysregulatin der mukosalen Immunantwort.

Eine erhöhte Produktion von Zytokinen und reaktiven Sauerstoffspezies, die von aktivierten Schleimhautmakrophagen produziert werden, schädigen das Darmepithel weiter und fördern den bakteriellen Translokationsprozess, wodurch dieser Teufelskreis der Darmentzündung aufrecht erhalten bleibt.

HOCl: Hypochlorid; IFN: Interferone; IL: Interleukine; LTB4: Leukotriene B4; NO: Stickstoffmonoxid; R.O.S.: reaktive Sauerstoffspezies; Th17: T-Helferzellen 17; TNF: Tumornekrosefaktor.

Autorin: PD Dr. Irina Blumenstein, Zentrum für Innere Medizin, Medizinische Klinik 1: Gastroenterologie und Hepatologie, Universitätsklinikum Frankfurt a.M. 


\section{Blickfang Pathogenese FokUs}

\section{Chronisch-entzündliche Darmerkrankungen}

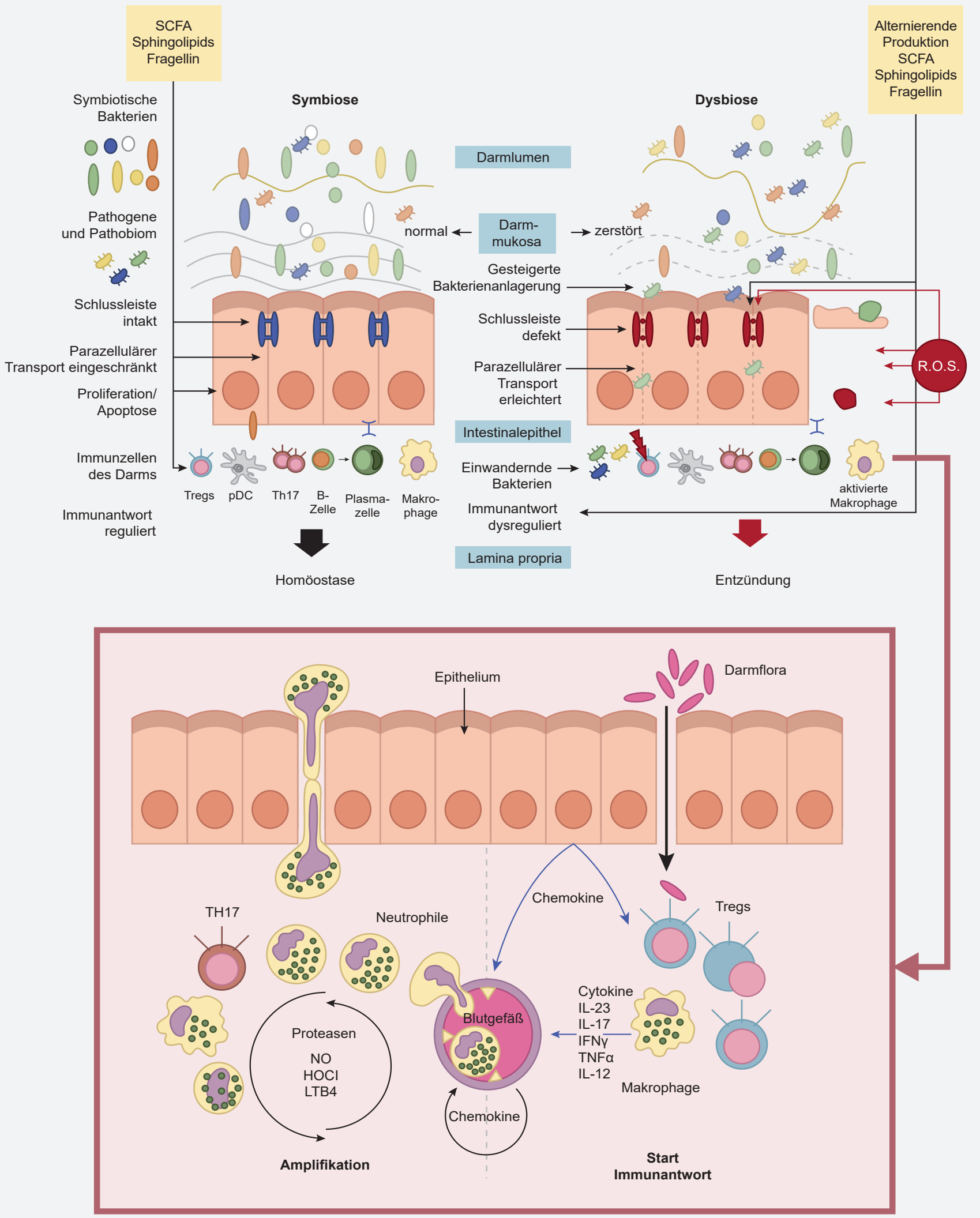

Grafik kombiniert und modifiziert nach

1 Aggeletopoulou I, et al.: The role of the gut microbiota in the treatment of inflammatory bowel diseases. Microbial Pathogenesis. 2019;137:103774.

2 Ramdan DS, Shanahan F: Fast Facts: Inflammatory Bowel Disease. 5th Edition, Health Press, Oxford, 2016. 


\section{Arzt-Patienten-Kommunikation}

\section{Mehr Humor im ärztlichen Berufsalltag}

Humor ist in aller Munde: bei Jan Böhmermann oder in der Serie «Die Anstalt», bei Otto Waalkes oder Loriot, bei Dr. Eckart von Hirschhausen oder Vince Ebert. Aber gibt es auch kompetente und humorvolle praktizierende Ärztinnen und Ärzte? Denn ein Blick in den ärztlichen Berufsalltag legt nahe, dass der Humor proportional zum Aufstieg auf der Karriereleiter häufig abnimmt.

Die Kombination aus humorvoll und kompetent kennt meist nur ein Kollege, der eine Kollegin hatte, die einen Kollegen kennt, der so ist. Aber selbst erlebt hat das noch niemand. Nur bei sich selbst natürlich - denn sind wir nicht alle unglaublich feinfühlig und haben bestechenden Witz?

Wenn wir uns jetzt unter den Kollegen umschauen, gibt es ein paar, mit denen können wir lachen. Ein Großteil taugt zur professionellen Zusammenarbeit und der Rest der Gauß'schen Verteilung ist gelinde gesagt eine Zumutung. Aber alle glauben bzw. sind überzeugt davon, in Sachen Kommunikation und Humor etwas drauf zu haben. Woran liegt das? Berufs- und Lebenserfahrung? Aus- und Fortbildungen in ärztlicher Kommunikation? Oder weil wir von Kindesbeinen an sprechen gelernt haben?

\section{«Humor ist der Knopf, der verhindert, dass uns der Kragen platzt.»}

\section{Joachim Ringelnatz}

Wenn wir ehrlich sind, haben die meisten von uns einen Strahlenschutz-Kurs, einen Abdomen-Ultraschall-Kurs oder einen BLS-Kurs besucht. Aber gilt das auch für einen Kurs in ärztlicher Kommunikation oder noch spezieller zum Thema «Humor in der Medizin»? Fehlanzeige! Dabei führen Ärztinnen und Ärzte gut

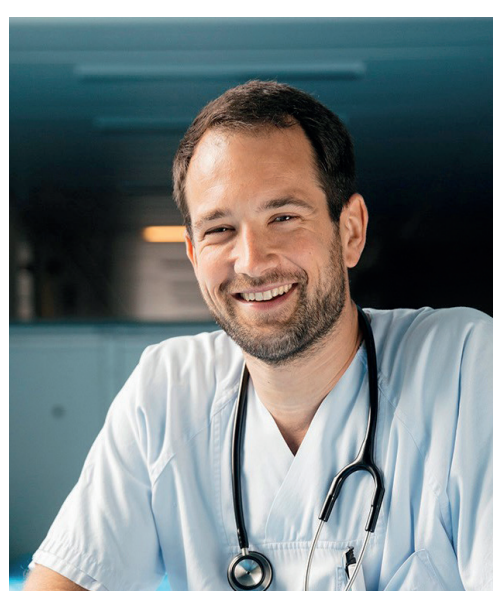

«Denn ein Blick in den ärztlichen Berufsalltag zeigt, dass der Humor proportional zum Aufstieg auf der Karriereleiter abnimmt.»
Aufmerksamkeit erhöht und er im medizinischen Kontext u.a. die kardiopulmonale Physiologie positiv beeinflusst, dass er ablenken kann, die Perspektive ändert und damit Ängste reduziert.

«Kommen eine Seele und ein Körper gemeinsam ins Krankenhaus. Sagt die Seele zum Körper: «Geh ruhig vor. Dich verstehen sie hier besser.» Ulrich Schaffer

Im medizinischen Kontext fehlt es nicht an Humor. Er bedient nur zu schnell Schenkelklopfer, wird zu plump oder grenzt unsere PeerGroups ab. Chirurgen und Anästhesisten können davon ein Lied singen. «Wie nennt man das grüne Tuch zwischen OP-Feld und Beatmung? Blut-Hirn-Schranke!» Das macht zwar kurzfristig Spaß, verbessert langfristig aber die Zusammenarbeit nicht.

Feinsinnig seinen humorvollen Fingerabdruck im stressigen Berufsalltag zu hinterlassen, das ist eine ärztliche Kunst, die begeistert. Mein Gegenüber humorvoll aufzuwerten, ein Ventil für Druck und Stress zu haben und sich selbst vor dem Ausbrennen zu bewahren, das alles kann ein spezielles Training bei «Arzt mit Humor» bewirken. Denn den Humor bringen Sie mit. Sie müssen ihn nur bewusst nutzen und die Situation nicht dem Zufall überlassen. Nicht aufgesetzt und künstlich, sondern authentisch und echt. Das sind dann Sie! Wenn die eigene Leistungsfähigkeit steigt, die Freude im Arztberuf wiederkommt und Humor zur Erhaltung der eigenen Gesundheit oder im Konflikt eine wirksame Ressource wird, dann haben Sie wohl ein Training von «Arzt mit Humor» besucht und es hat Sie überzeugt.

Das nächste Training «Humor auf Rezept? Die Dosis bringt den Erfolg!» für Ärztinnen und Ärzte findet vom 06./07.11.2020 in Leipzig 200000 Gespräche im Laufe ihrer Karriere. In einem Bereich der hochsensibel für Missverständnisse, Kompetenzgerangel und anstrengende Patienten ist. Nicht schlimm, das kann man ändern. Die Initiative «Arzt mit Humor» hat sich auf die Fahnen geschrieben, den Humor auch im Krankenhaus oder der Arztpraxis salonfähig zu machen. Denn die Gauß'sche Verteilung der Kolleginnen und Kollegen mit Humor entsteht nicht, weil das Gegenüber keinen hat, sondern weil es Ihren Humor nicht teilt oder eben einen anderen hat. Dasselbe gilt übrigens auch für Patienten, ihre Angehörigen und das Pflegepersonal.

Wir wundern uns manchmal, wieviel «Arzt» ein Patient verträgt? Weg von der rein medizinischen Sichtweise interessiert mich, wieviel Humor haben meine Patienten, meine Kollegen, das Pflegepersonal noch bzw. wieviel geht noch? Oder warum ist der Humor abhandengekommen?

Oder kann Humor meinem Problem schaden? Man weiß, dass sich in entspannten Situationen besser lernen lässt, dass Humor die statt. Bereits am 08. Septemer bieten die Veranstalter ein OnlineLive Seminar zum Thema an, für das man sich auf der Homepage schon jetzt voranmelden kann. Weitere Informationen unter www.arztmithumor.de.

Dr. Christoph Krause ist Anästhesist am Universitätsklinikum Leipzig und fährt als Notarzt durch die Stadt. Vor Jahren hat er zusammen mit dem «Deutschen Institut für Humor» die Initiative «Arzt mit Humor» ins Leben gerufen, weil der Berufsalltag nicht immer die wahre Freude war, wenn es um den Umgang zwischen der Ärzteschaft, den Studierenden, dem Pflegepersonal, den Patienten und den Angehörigen ging. Wohl wissend, dass es im Gesundheitswesen Faktoren gibt, die den Idealismus verschrecken, hält er an der Vision fest, gute ärztliche Kommunikation und Hochleistungsmedizin miteinander zu verbinden.

Kontaktadresse: Dr. Christoph Krause, Deutsches Institut für Humor $(\mathrm{DIH})^{\circledR}$, Feuerbachstraße 26, 04105 Leipzig, Deutschland, christoph. krause@arztmithumor.de. 